\title{
CAPÍtULLO X
}

\section{INFLUENCIA DE LOS VIDEOJUEGOS EN EL COMPORTAMIENTO DE LOS ADOLESCENTES}

\section{Dorkys Coromoto Rojas Nieves.}

Doctora en Ciencias Gerenciales. Universidad Dr. Rafael Belloso Chacín Investigadora activa de la Alianza de Investigadores Internacionales S.A.S. ALININ, perteneciente al grupo de investigación GICUINT. correo dorkysr@gmail.com ORCID ID: 0000-00017913-6549.

\section{Yamarú del Valle, Chirinos Araque.}

Doctora en gestión de la innovación, Universidad Dr. Rafael Belloso Chacín. Investigadora Asociada categorizada por Colciencias. Docente- investigadora del grupo: GORAS. Universidad Católica Luis Amigó, Medellín, Colombia. yamaru.chirinosar@amigo.edu.co; urumay78@gmail.com. ORCID ID. 0000-0003-04719859.

\section{Gustavo Adolfo Moreno López.}

PhD (c) en Administración de la Universidad de Medellín-Colombia,. Rector de la Institución Universitaria Marco Fidel Suárez. Integra el grupo de Investigación Grieso de la Institución Universitaria Marco Fidel Suárez, Bello - Antioquia. rectoria@iumafis.edu.co ORCID: 0000-0002-4094-0940.

\section{Nataliya Barbera de Ramírez.}

Doctora en Planificación y Gestión del desarrollo Regional de la Universidad del Zulia. Docente investigador del Departamento Currículo Común Unisinú, Facultad de Ciencias Humanas, Arte y Diseño, Universidad del Sinú, Montería. nataliaberbera@unisinu.edu.co. ORCID ID: http://orcid.org/0000-0002-4566-5052.

\section{Oditza Nacrina Bracho Vega.}

Doctorado en Educación UNERMB Año 2016. Docente Asociado de la UNERMB, investigadora activa de la Alianza de Investigadores Internacionales S.A.S. ALININ, perteneciente al grupo de investigación GICUINT.ORCID https://orcid.org/0000-00034685-2498 correo oditzabrachovega@gmail.com

\section{Resumen}

El estudio tuvo como propósito determinar Influencia de los videojuegos en los adolescentes en el comportamiento de los adolescentes del 5to año de bachillerato Unidad Educativa Alfredo Armas Alfonzo, ya que hoy en día este tipo de entretenimiento se ha vuelto muy popu- 
lar. Metodológicamente, el tipo de investigación fue descriptiva; el diseño de campo, no experimental, transeccional; la población estuvo conformada por los 55 estudiantes del 5to año de bachillerato de la unidad educativa Dr. Jesús María Portillo. Se diseñó una entrevista, la cual se aplicó vía telefónica y los resultados se vaciaron en una matriz para su posterior análisis. Se llegado a la conclusión que los adolescentes que cursan el 5 to año no presentan cambios negativos en su comportamiento a consecuencia del uso de los video juegos, aun cuando sienten predilección por los videojuegos de acción los cuales tienen un alto contenido de violencia sangrienta, tampoco muestran signos de aislamiento debido a que se evidenció que comparten este gusto con amigos y conocidos.

Palabras clave: adolescentes, comportamiento, jugador, videojuegos.

\title{
INFLUENCE OF VIDEO GAMES ON ADOLESCENTS ON THE BEHAVIOR OF ADOLESCENTS
}

\begin{abstract}
The purpose of the study was to determine the influence of video games in adolescents on the behavior of adolescents in the 5th year of high school in the Alfredo Armas Alfonzo Educational Unit, since nowadays this type of entertainment has become very popular. Methodologically, the type of research was descriptive; the field design, non-experimental, transectional; The population was made up of 55 students of the 5th year of high school from the educational unit Dr. Jesus Maria Portillo. An interview was designed, which was applied via telephone and the results were emptied into a matrix for later analysis. It was concluded that adolescents who are in the 5th year do not present negative changes in their behavior as a result of the use of video games, even though they have a predilection for action video games which have a high content of bloody violence, neither do they show signs of isolation due to the fact that it was evident that they share this taste with friends and acquaintances.
\end{abstract}

Keywords: adolescents, behavior, gamer, videogames.

\section{Identificación del Proyecto}

Valores éticos-morales pilares en la formación de competencias investigativas. Financiado por la Alianza de Investigadores Internacionales S.A.S.

\section{Introducción}

Es indiscutible que en la actualidad se vive en una época regida por grandes cambios, muchos de los cuales están ligados a los avances tecnológicos, los mismos llegaron para 
cambiar la forma en que los individuos se movilizan, la forma en la que obtienen la información, la forma en que se entretienen, hasta la forma en que se relacionan. El área donde estos cambios se han visto más acentuados es en el campo del entretenimiento con la aparición y desarrollo de los videojuegos.

$\mathrm{Al}$ crecer en un mundo digitalizado los adolescentes han convertido a los videojuegos en su entretenimiento favorito, ya que pueden usar varios aparatos a su disposición para jugar como tablet, consola, laptop, pc y hasta un teléfono; los mismos hacen muy fácil estar en contacto con el videojuego, pudiendo dedicar horas en este entretenimiento y en algunos casos dejan de lado actividades como hacer deporte, estar al aire libre o socializar con amigos y familiares solo por el placer que obtienen al jugar videojuegos. Para autores como García-Naveira et al., (2018), los videojuegos son "juegos electrónicos que se visualizan en una pantalla" (p. 2), y que están siendo impulsados por la conectividad a la red y los avances tecnológicos.

Una de la poblaciones que más hacen uso de estos videojuegos son los adolescentes, ellos al estar en un periodo de crecimiento y desarrollo, en la transición entre la niñez y la adultez, le ocurren cambios significativos físicos, mentales, sociales y hasta económicos, además se les considera altamente vulnerables ante las presiones de la sociedad, es por esto que al ser los videojuegos una representación de la sociedad podrían verse confundidos y tratar de imitar situaciones que en éstos se presentan, esta preocupación es real, ya que los contenidos de algunos de estos videojuegos muestran violencia, guerra, terror, peleas o sangrientas confrontaciones, aun cuando existen los videojuegos educativos.

Muchos son los investigadores que hacen hincapié en las consecuencias negativas del uso de los videojuegos, los responsabilizan del sedentarismo, el aislamiento social, bajo rendimiento escolar, la agresividad, el egoísmo y la impulsividad en los adolescentes. Sin embargo, existen otros quienes piensan que su uso tiene sus beneficios, ya que proporciona un sentido de dominio y control, no es solo una forma de entretenimiento sino también de aprendizaje, puede disminuir la intensidad de conductas problemáticas, permiten el logro de metas concretas, entre otros.

Lo anteriormente planteado ha dado origen a diversas investigaciones como las realizadas 


\section{Fundamentación teórica}

\section{Videojuegos definición}

La Real Academia de la Lengua Española considera que es "un dispositivo electrónico que permite, mediante mandos apropiados, simular en las pantallas de un televisor o de un ordenador", por su parte Vigueras (2001), manifiesta que "son medios de entretenimiento con características propias que tiene diferencias y similitudes con otros medios, su narrativa es cautivadora y posee representaciones simbólicas, tienen la propiedad de lograr una concentración absoluta en los jugadores”. Márquez (2001), es más preciso cuando dice que los videojuegos son todo tipo de juegos digitales interactivos, con independencia de su soporte (ROM interno, cartucho, disco magnético u ópticos, on-line) y plataformas tecnológicas (máquinas de bolsillos, videoconsolas conectadas al televisor, teléfonos móviles, máquinas recreativas, microordenador, ordenador de mano, video interactivo).

La definición de Estallo (1995), es más completa, ya que él considera que los videojuegos son una forma de ejercicio cognitivo y gimnasia mental, realizado por el usuario para satisfacer necesidades intrínsecas y disfrutar del tiempo del tiempo de ocio jugando con objetos interactivos que permiten: alcanzar un determinado objetivo, un estado de ánimo, algunas metas, ejecutar acciones fantásticas y adquirir algunos niveles de control y destreza.

Provenzo (1991), manifiesta que se han desarrollado y crecido solo porque vivimos en una sociedad de consumo, sin embargo, recalca que permite a los usuarios conectarse a través de poderosas redes de información, comunicación, ocio y entretenimiento,

Es importante señalar que muchos son los autores que han dado una definición de los videojuegos, sin embargo, no se han puesto de acuerdo en lo que significa el termino, ya que cada uno tienen una idea de lo que es y hacen énfasis en un determinado aspecto o elemento, esto ha impedido un concepto homogéneo.

Lo que si queda claro es que su uso se ha incrementado considerablemente y aunque esta tecnología forma parte del ocio y entretenimiento están siendo incluidos en otras áreas, como el educativo. 
por el departamento de Medicina de Yale, el cual encontró que entre el 7\% y el 11\% de los adolescentes que utilizan los videojuegos tienen problemas reales en su comportamiento afectando directamente sus relaciones familiares, sociales y escolares; este porcentaje de adolescentes presentaban problemas para dejar de jugar, tensión que solo era aliviada al jugar, etc; por otro lado una pequeña porción de estos adolescentes evidenciaban una tendencia a la violencia, al uso de drogas o fumar.

La Universidad Estatal de Iowa también realizó investigaciones en esta área, descubriendo que solo el $10 \%$ de los adolescentes que les gusta jugar videojuegos presentan signos de depresión, ansiedad, bajo rendimiento escolar y no se adaptan socialmente. (Diez et al., 2002).

Por estas razones los padres y familiares de los adolescentes han comenzado a preguntarse qué tan beneficioso o perjudicial es el uso de los videojuegos, cuáles son los efectos o posibles consecuencias en el comportamiento de sus hijos, ya que su uso se ha generalizado por la facilidad de hacerlo en cualquier aparato portátil (teléfonos, tablets o laptop) que los muchachos puedan poseer. Ante lo anteriormente planteado es pertinente formular la siguiente pregunta de investigación ¿Cómo influyen los videos juegos en el comportamiento de los adolescentes?

En la actualidad existe una gran polémica sobre los beneficios o no, de la utilización de los videojuegos, existe evidencia que el aislamiento social, el sedentarismo y hasta el bajo rendimiento escolar pueden ser causados por un exceso de uso de los videojuegos, pero también es cierto que los jugadores aprenden a desarrollar estrategias, les es menos traumático el perder, así como les permite de una manera más fácil la creación de soluciones a los problemas, entre otros, sin embargo persiste la preocupación por los aspectos negativos.

Estudiar el comportamiento que tienen los adolescentes ante el uso de este instrumento de entretenimiento se hace importante y justificado porque estos individuos están en pleno desarrollo de su personalidad, y por tanto son muy susceptibles al tipo de información a la cual se enfrentan, además, es en esta etapa donde se adquieren los hábitos de vida saludables. Ante lo cual se desarrolló como objetivo de investigación establecer cómo influyen los videojuegos en el comportamiento de los adolescentes. 


\section{Características de los videojuegos}

Los juegos presentan una serie de características que también están presentes en los videojuegos autores como Huizinga (2000), Newman (2004), y Wolf (2001), muestran en la siguiente tabla las que consideran son sus características:

Tabla 1. Características videojuegos

\begin{tabular}{|l|l|}
\hline & Se practica con la finalidad de satisfacer, dentro de la propia \\
& libertad del jugador. \\
& El juego se desarrolla dentro de unos límites fijados por él, que \\
determinan su objetivo. & Finalizado, perdura en la memoria junto a una valoración perso- \\
Huizinga (2000) & Posee una serie de reglas que el jugador debe cumplir para fina- \\
& lizar el juego. \\
Cada jugador posee unas peculiaridades que ocasionan un deter- \\
minado ritmo y armonía. \\
Su resolución es producto de la presión soportada por el jugador \\
durante el transcurso del juego.
\end{tabular}

Fuente: elaboración propia (2021).

Al estudiar a los diferentes autores se puede afirmar que los videojuegos comparten características con los juegos tradicionales, pero ante los avances tecnológicos con sus efectos, imágenes, sonido, capacidad de memoria, entre otros los hacen más atractivos y competitivos. 


\section{Clasificación de los videojuegos}

Es importante entender y saber cuáles son los tipos de videojuegos a los que tienen acceso la población, es así que Hernández (2015), desarrollo una de las clasificación más completas luego de hacer una revisión de las clasificaciones hechas por otros investigadores y en ella se cumplen con cuatro condiciones fundamentales: 1.- se basa en el gameplay; 2.- criterios de clasificación común a otros autores e industria; 3.- omisión de géneros prescindibles que pueden ser incluidos en otros; 4.- evitando el solapamiento de las categorías planteadas. Quedando construida de la siguiente manera:

*Acción: se trata de videojuegos con un desarrollo lineal, basados en la habilidad, pericia, precisión y tiempo de reacción del jugador para avanzar en contexto de combate, superación de obstáculos o peligro.

*Aventura: basado en la recreación de algún tipo de aventura o trama con un argumento extenso y enrevesado, en la que el personaje deberá superar diversas pruebas y situaciones que se van sucediendo a través de determinadas acciones, las cuales le permitan avanzar y cumplir el objetivo final.

*Simulaciones: videojuegos en los que el sujeto emula situaciones reales de la forma más fiel posible, a modo de reproducción de una experiencia objetiva, lo que requiere de ciertos conocimientos específicos sobre el manejo de la acción a simular.

*Deportes: videojuegos basados en la recreación de algún deporte como futbol, baloncesto, golf, rugby, etc. Las mecánicas de los juegos suelen basarse en las reglas reales de los deportes, pero en ocasiones incorporan añadidos u otros modos de jugabilidad.

*Conducción: el género se basa en el control y dirección de vehículos con la finalidad de culminar determinados objetivos. La conducción está basada en la realidad, pero los vehículos no simulan las físicas reales.

*Estrategia: videojuegos basados en el control y la organización de determinadas 
situaciones económicas, empresariales, sociales, etc, a través de la manipulación de personajes, objetos o datos con el fin de lograr determinados objetivos.

*Rol: este género se basa en juegos homólogos de mesa. El sujeto asume el papel de uno o varios protagonistas, situados en mundos fantásticos y en los que debe mejorar sus habilidades a través de la interacción con otros personajes y el entorno.

*Shooter: el género está basado en videojuegos en los que el jugador, en primera o tercera persona, tiene que utilizar material armamentístico para derribar elementos y personajes. Los shooter basan su éxito en los modos competitivos donde varias personas pueden participar de forma online.

*Arcade: incluye a todos los videojuegos clásicos de las máquinas recreativas. Estos juegos se caracterizan por tener un ritmo rápido, con tiempos cortos, creciente dificultad y basados en una jugabilidad sencilla, que permite al jugador avanzar sin necesidad de recrear comportamientos estratégicos.

*Casual: videojuegos basados en temáticas de entretenimiento o educación que están destinados a jugadores no habituales que buscan una distracción diferente. Están basados en reglas simples y no requieren de gran dedicación ni compromiso.

\section{Ventajas y desventajas del uso de los videojuegos}

Los videojuegos llegaron para quedarse en la vida de las personas y hoy en día es una de las maneras de entretenerse más populares, son muchos los prejuicios negativos que se tiene sobre su uso, debido a que se le responsabiliza por el aumento en la agresividad, el aislamiento de quienes los utilizan, sin embargo existen otras que piensan que tiene su lado positivo consideran que propician un sentido de dominio y control, su lado estratégico es desarrollado, estimula el aprendizaje, por eso autores como Betancourt (2015), consideran que existen una serie de ventajas y desventajas que se presentan en la siguiente tabla: 
Tabla. 2 Ventajas y desventajas de los videojuegos

\begin{tabular}{|c|c|}
\hline Ventajas: & Desventajas: \\
\hline $\begin{array}{l}\text { - Fomentan la motivación: mucho de ellos } \\
\text { tienen una atractiva interfaz y navega- } \\
\text { ción que permite estimular varios senti- } \\
\text { dos al mismo tiempo. } \\
\text {-- Tienen gran interactividad: lo que permi- } \\
\text { te que el usuario sea un sujeto activo y } \\
\text { participativo mientras lo usa. } \\
\text {-- Facilita la atención a la diversidad: per- } \\
\text { mite establecer varios ritmos de aprendi- } \\
\text { zaje y la facilidad de adaptar el nivel de } \\
\text { contenidos según las necesidades de cada } \\
\text { uno. } \\
\text {-- Estimula la capacidad de racionamiento y } \\
\text { discernimiento. } \\
\text {.- Permite la interdisciplinariedad: en un } \\
\text { solo juego se pueden encontrar desarrollo } \\
\text { de varias áreas. } \\
\text {-- Permite un sistema de evaluación que } \\
\text { genera la sana competencia. } \\
\text {.-Favorece la organización espacio- } \\
\text { temporal. } \\
\text {-- Ayuda en el desarrollo de destrezas bási- } \\
\text { cas como la rapidez de reflejos y la me- } \\
\text { moria. }\end{array}$ & $\begin{array}{l}\text {.- Puede generar adicción. } \\
\text {.- El uso excesivo puede generar rechazo ha- } \\
\text { cia otros medios didácticos como libros, } \\
\text { CD, etc. } \\
\text {.- Puede causar conductas violentas si el re- } \\
\text { curso utilizado se basa en este tipo de com- } \\
\text { portamiento. } \\
\text {.- Quienes juegan se desconectar del mundo } \\
\text { real y pueden dejar de existir en el tiempo y } \\
\text { el espacio; el afuera y la realidad exterior. } \\
\text {.- Los videojuegos evitan la comunicación y } \\
\text { la familia. } \\
\text {.- Restan tiempo a las tareas escolares. } \\
\text {.- Generan estrés y fatiga ocular. } \\
\text {-- Dan lugar a dolores musculares y articula- } \\
\text { res. }\end{array}$ \\
\hline
\end{tabular}

Fuente: elaboración propia (2021) basado en Betancourt (2015).

Para León y López (2003), la aparición de los videojuegos como fuente de entretenimiento de niños y adolescentes ha generado una gran controversia entre quienes piensan que tienen ventajas como que favorece el autocontrol, reduce las conductas problemáticas, potencia el aprendizaje, desarrolla la coordinación ocular y manual, además potencia el aprendizaje; y los que piensan por el contrario que tiene serias desventajas como restan tiempo al estudio, así como a otras actividades de ocio y educativas, favorecen conductas agresivas, limitan el desa- 
rrollo de habilidades sociales y fantasía, así como el consumo y gasto de dinero.

$\mathrm{Al}$ analizar a los autores se puede decir que en su mayoría los investigadores están de acuerdo en que los videojuegos tienen una serie de ventajas y desventajas, en las cuales casi todos coinciden, pero además se encuentran altamente preocupados por el bienestar social y psicológico del segmento que más consume este tipo de entretenimiento que son los adolescentes, los cuales al estar en una etapa de desarrollo psíquica y físicamente son, desde su punto de vista, más vulnerables.

\section{La adolescencia}

La adolescencia es un periodo de cambios bio-psíquico-social por los cuales transitan los seres humanos, ocurre entre la niñez y la adultez, se estima que ocurre entre los 10 y 20 años según Silva (2017), sin embargo, esto puede variar de acuerdo con el autor que se consulte, por ejemplo, la Organización Panamericana de la Salud (2005), la ubica entre los 10 y 19 años, es en esta etapa cuando se alcanza la madurez biológica y se busaca alcanzar la emocional y social. Es en este periodo cuando se comienzan a tomar decisiones y a asumir sus consecuencias, se han malas o buenas, se conocen las fuerzas internas, Coon (citado por Luzuriaga, 2013), establece que esta etapa está determinada por 4 factores la dinámica familiar, la experiencia escolar, las condiciones económicas y políticas del momento, por último, el marco cultural que ha estructurado el ambiente social para la puesta en práctica de normas y límites.

Esta es una etapa de confusión para el adolescente, porque el niño llega a ella sin saber cuánto dura, ni que debe o no debe hacer, la sociedad y la familia contribuyen a este estado de indeterminación, al no tener claro cómo tratarlos, ya que los adolescentes se encuentran en un punto intermedio entre lo que hace un niño y lo que hace un adulto. Hasta el famoso psicólogo Freud (citado por Madrigales 2012), consideraba que los cambios psicológicos presentados en esta etapa son importantes, pero también las transformaciones de la personalidad.

Madrigales en su investigación a firma que la adolescencia es un periodo de cambios biopsicosociales donde el individuo tienen una búsqueda de su identidad, lo cual lo hace susceptible a cambiar repentinamente su manera de actuar, por ejemplo está a favor de la justicia o en 
contra de ella, pero si puede conseguir las cosas de la manera fácil no duda en hacerlo, solo por los valores y normas que trae del seno familiar, tiene gran interés en lo que ocurre fuera del contexto familiar, lo que lo hace vulnerable a las adicciones.

\section{Etapas de la adolescencia}

\section{Adolescencia temprana}

Luzuariaga (2012), manifiesta que esta etapa comienza entre los 10 y 12 años, se presentan los primeros cambios físicos y se da inicio a la madurez psicológica, pasando del pensamiento concreto al abstracto, tratan de crear sus propios criterios, socialmente se aleja de casi cualquier relación con niños, pero no es aceptado por los adolescentes de mayor edad, lo que provoca una desadaptación social, incluso dentro de su familia, esto puede causar alteraciones emocionales como depresión y ansiedad, que influyen en su conducta, contribuyendo a su aislamiento.

A estos cambios hay que agregarles los del entorno escuela, maestros, compañeros, sistema educativo y responsabilidades. Todo esto se denomina crisis de entrada en la adolescencia. Papalia (2001), considera que presencia de la familia (padre, madre, tutor), se convierte en un factor importante de como el adolescente vive esta etapa, ya que el apoyo y la autoridad le ayudaran a estructural su personalidad, así como a superar su ansiedad.

\section{Adolescencia intermedia}

Para Luzuariaga (2012), esta etapa se inicia entre los 14 y 15 años, aquí se alcanza cierto grado de aceptación y adaptación de si mismo, se comienza a tener un parcial conocimiento y percepción con respecto al potencial que posee, aquí ya tiene integración con su grupo, esto le da cierta seguridad y satisfacción, además de establecer amistades. Manifiesta Papalia (2001), que es en esta etapa cuando el adolescente empieza a adaptarse a otros adolescentes, por lo tanto, integra grupos y se les más sencillo integrase a ellos, lo que resuelve parcialmente el rechazo de los padres, por lo que deja de admirar el rol de los padres. 


\section{Adolescencia tardía}

Esta etapa comienza entre los 17 y 18 años, según Luzuariaga (2012), quien también manifiesta, que es aquí cuando disminuye la velocidad de crecimiento y se comienza a recuperar la armonía en la proporción de los diferentes segmentos corporales, lo que va dando seguridad y ayudan a superar la crisis de identidad, se tiene más control de las emociones, poseen más independencia y autonomía. Para Papalia (2001), existe todavía un cierto grado de ansiedad al empezar a percibir las presiones de las responsabilidades presentes y futuras, propias o de la familia, para contribuir con la economía, aunque todavía no alcanza la madurez ni tiene los elementos de experiencia para desempeñarlas.

Entonces se puede concluir, apoyados en lo expuesto por Silva (2007), que un adolescente es aquel individuo que vive una serie de transformaciones personales emocionales, corporales y sociales, que están en la búsqueda de su identidad, de sus potencialidades, que les permita en relación con otros construir una historia personal significativa.

\section{Tipos de conducta}

Toyo (2013), señala que la conducta está determinada por múltiples factores que van desde lo genético o hereditario, los cuales hacen referencia a la conducta innata (instintiva) que existe desde que nacemos. Pasando por los situacionales o del medio, que son las conductas concretas que se dan ante una situación determinada (aprendida). Hasta los orgánicos, que están representados por las acciones conjuntas de los sistemas nerviosos y endócrino cuya actividad sienta las bases orgánicas de la conducta y los ambientales donde la conducta está condicionada por el entorno que los rodea, como estímulos externos.

Esta autora considera que los tipos de conducta son la agresiva, la pasiva, la asertiva, la prosocial y las motivadas aprendidas, a las cuales define de la siguiente manera:

- La conducta agresiva: se considera que es la forma de expresión de los sentimientos, creencias y opiniones que pretenden hacerla valer, pero atacando o no considerando la sensibilidad o respeto hacia los demás. 
- La conducta pasiva: los demás se aprovechan del individuo fácilmente, además que fomenta esa actitud, suelen presentar sentimientos de inseguridad e inferioridad que se refuerzan siempre que entra en contacto con una persona agresiva. Se encuentra enfadado consigo mismo porque no hace o no puede hacer nada para que no se aprovechen de él, es tímido y reservado, no sabe aceptar cumplidos, no tiene mucha energía y entusiasmo.

- La conducta asertiva: es la habilidad de trasmitir y recibir los mensajes y sentimientos, creencia y opiniones de los demás y las propias de manera honesta, oportuna y respetuosa. Le es importante lograr una comunicación satisfactoria hasta donde el proceso humano lo amerite.

- La conducta prosocial: Eisenberg (citado por Toyo 2013), considera que la conducta prosocial se define como las acciones que tienden a beneficiar a otros, sin que se prevean recompensas externas. Esta conducta aparece en la etapa pre-escolar, sigue desarrollándose ya entrada la adolescencia y posteriormente, son las situaciones, las normas familiares y culturales las que deciden que conducta se considera socialmente apropiadas.

- Las conductas aprendidas: en este caso no existe un objetivo inmediato a alcanzar, pero a través de reflejos condicionado, a través de experiencias precedentes, se introducen determinadas conductas que se necesitan reproducir. Algunos de los logros alcanzados se convierten en reforzadores positivos, llamados recompensas, que implican la obtención de un placer, entre ellos encontramos la necesidad de seguridad, de aprobación, de interrelaciones, de amor, de aspiraciones, de poder, entre otros.

Al ser la adolescencia la etapa de la vida de los seres humanos donde se experimentas los cambios de conducta más significativos, se puede observar que en su mayoría ellos mantienen una actitud distante y menos afectuosa hacia su familia, quieren cambiar las normas, permanecen absorto mucho tiempo, eluden las responsabilidades impuestas, tienen una actitud negativa hacia las personas y en muchos casos hacia ellos mismos, muestran más interés hacia sus amigos, toman sus propias decisiones siguiendo sus propios criterios, aunque no le guste a los que 
están a su alrededor, su rendimiento escolar se ve afectado en algunos caso por falta de motivación, de forma brusca cambian emocionalmente. Estos comportamientos propios de la adolescencia preocupan porque los convierten en individuos vulnerables y con dificultades para la interacción y adaptación social.

\section{Metodología}

La investigación se desarrolló bajo un enfoque cualitativo con alcance descriptivo, citando a Hurtado (2000), "la investigación descriptiva tiene como objetivo central, lograr la descripción o caracterización del evento de estudio dentro de un contexto particular" (p. 223). Con un diseño no experimental que, para Hernández et al., (2014) son aquellas donde no existe la manipulación deliberada del fenómeno que es observado y los que se busca es describirlo, analizarlo y explorarlo.

Como instrumento de recolección de datos se utilizó una entrevista semiestructurada donde se le preguntó a un grupo de 55 estudiantes adolescentes pertenecientes al 5 to año de bachillerato de la unidad educativa Dr. Jesús María Portillo, la misma fue realizada vía telefónica debido a la contingencia ocasionada por la pandemia del COVID-19, sus respuestas serán apoyada con documentos que abordan el tema sobre la influencia que tiene los videojuegos en los adolescentes, obtenidas de diferentes base de datos indexadas de alto impacto tales como Scopus, Elsevier Dialnet, Google Scholar,

\section{Resultados}

Briceño et al., (2018), en su investigación afirma de manera conducente que ante la era digital en la cual vive la sociedad, hoy en día una sociedad tecnológica "donde los hábitos y estilos de vida se han visto transformados por el desarrollo constante e imparable de las tecnologías e Internet" (p. 168), por lo tanto, no es de sorprender que ante la pregunta a los adolescentes sobre si les gustaba jugar videojuegos el 95\% respondiera que si le gustaba y si los juega.

Por su parte, cuando se les pregunto por el tipo de videojuego que preferían jugar el 
$62,50 \%$ respondió que videojuegos de acción, un 25\% videojuegos de rol, mientras que un $12,50 \%$ de estrategias, lo que deja en evidencia el favoritismos de los adolescentes del 5 to año de bachillerato de la Unidad Educativa Dr., Jesús María Portillo por los videojuegos de acción, en cierta medida esto es preocupante, debido a que estos juegos muestran escenas sangrientas, altamente violentas y ponen al jugador en la posición de asesinar personas ficticiamente, hay que recordar que los adolescentes son individuos en crecimiento, no solo físico sino también mental, están en una etapa de trasformación, que según el psicólogo Freud (citado por Madrigales 2012), son importantes, ya que impulsan el desarrollo de la personalidad, por lo tanto pudieran verse afectados negativamente en su desarrollo, al tener acceso a este tipo de videojuego de manera indiscriminada.

Ahora bien, ante la pregunta con qué frecuencia utilizan los videojuegos un $43,75 \%$ de los estudiantes respondieron que lo jugaban constantemente, mientras que otro $43,75 \%$ que lo utilizan de vez en cuando y un $12,50 \%$ que pocas veces, el jugar con tanta frecuencia ocasiona que los adolescentes pasen mucho más tiempo encerrados o sentados, en vez realizar actividades al aire libre, en especial actividades deportivas, tan necesarias para mantener una vida saludable, por lo tanto el sedentarismo, la obesidad pueden ser consecuencias nefastas de la alta frecuencia en el uso de estos juegos. Para Moncada y Chacón (2012), esta cantidad de tiempo que los estudiantes pasan frente a la pantalla jugando no cambiara en los próximos años, debido al acceso cada vez más fácil y frecuente a dispositivos como laptops, teléfonos digitales, consolas, entre otros.

A la pregunta cuando utilizas los videojuegos un 18,75\% de los adolescentes respondió que los días de clases, un $68.75 \%$ los fines de semanas y un $12,50 \%$ todos los días, lo que permite asumir que un alto porcentaje respeta los días de escuela a la hora de decidir en qué momento jugar, evidenciado un control parental que busca que estos jóvenes se concentren en sus estudios, por lo menos los días de clase. Esto es relevante porque como manifiesta Pérez (2013), los jóvenes están viviendo en una era digital que ha condicionado su manera de actuar, de relacionarse y hasta comunicarse, y no creen que estén haciendo un uso abusivo de esta tecnología. Es por ello como sostiene Betancourt (2015), el número de horas que le dedican a los videojuegos si son excesivas puede causar adicción, restarle tiempo con la familia y para hacer sus tareas escolares, lo que afectaría su rendimiento escolar, es así que recomienda que las horas 
que los adolescentes pasan con los videojuegos debe ser controladas, para que su uso sea el más adecuado posible.

En cuanto al comportamiento que tienen los estudiantes del 5 to año de bachillerato no presentan las características de un estudiante pasivo que tal como lo manifiesta Toyo (2013), es alguien que no sabe expresar sus emociones y no hace valer sus opiniones, por naturaleza son tímidos, reservados e introvertidos, ya que cuando se les pregunto a estos estudiantes si podían expresar sus opiniones y emociones con facilidad el $87,5 \%$ contesto que siempre lo hacen que les gustaba compartir con sus compañeros y amigos, además sus profesores y padres respetaban sus opiniones orientándoles cuando consideraban no tenían la razón, mientras que solo el $12,50 \%$ manifestó que nunca que les era difícil poderlo hacer en especial por que no confiaban en las personas o se sentían discriminados. Ante lo manifestado por la mayoría se podría decir que este no es un grupo pasivo.

En cuanto a la pregunta haces valer tus derechos tus opiniones y derechos usando la agresión los adolescentes en un su mayoría 72 \% respondieron que no y dieron como razón que en sus casas les habían enseñado que ese no es un comportamiento aceptable y que se debe intentar hablar sin llegar a los gritos, amenazas y golpes, por su parte el resto de los 55 estudiantes aunque no manifestó abiertamente que se iría a los gritos y golpes para hacer valer sus derechos u opiniones manifestaron que de tener que hacerlo lo harían. Toyo (2013), considera que un adolescente agresivo mostrará sus emociones y sentimientos sin tener ninguna consideración, respeto o sensibilidad para con los que le rodea, llegando incluso a la agresión fisca con tal de hacer valer lo que él piensa, característica que pocos de los estudiantes encuestados presentan.

Cuando se les dio a escoger qué preferían hacer en su tiempo libre las respuestas fueron variadas, sin embargo, la mayoría $(37,50 \%)$ se inclinó por los videojuegos, el $25 \%$ gustan de ver televisión, un $18,75 \%$ prefieren hacer deportes, el $12,50 \%$ por su parte le gusta ir al cine, un $6,25 \%$ prefieren leer un libro, esto evidencia que los videojuegos son el entretenimiento preferido por los adolescentes, que cada día son más populares, por tanto hay que estar atentos a que no abusen de su uso, ya que como dice Betancourt (2015), pueden presentarse ventajas como facilitar la atención a la diversidad, estimula los sentidos, favorece la organización espa- 
cio-temporal, pero también desventajas como adicciones, rechazo a los libros, restan tiempo a las tareas escolares, entre otras.

Una pregunta importante que se les hizo fue si eran capaces de hacer lo que hacían en los videojuegos en el mundo real, solo un pequeño grupo de ellos respondió que si lo haría, sin embargo por su tono de voz y su risa, pareciera que solo lo dijeran para impresionar, sin embargo, un alto porcentaje $(81,25 \%)$ respondieron que nunca lo harían que ellos sabían diferenciar una fantasía de la realidad y solo juegan por diversión. Esto permite afirmar que los sujetos investigados no presentan conflictos en identificar la realidad de la fantasía.

Los hallazgos que se desprendieron de la aplicación de la entrevista permiten afirmar que para este grupo de estudiantes la influencia de los videojuegos es positiva, en especial porque se evidencio un control parental significativo, lo que es muy importante en la regulación en el uso de estos juegos.

\section{Conclusiones}

En la actualidad sería absurdo pensar que los adolescentes no estarían interesados en jugar videojuegos y esto fue evidente al abordar a los estudiantes del 5to año de bachillerato de la Institución educativa Dr. Jesús María Portillo cuando en su totalidad los mismos manifestaron jugar algún tipo de videojuegos y le dedican una cantidad considerable de horas, las cuales no son más debido al control que ejercen sus padres, quienes impiden ocupar a los adolescentes más horas de su tiempo en eso, condicionando su uso en su gran mayoría a los fines de semanas cuando no acuden a la institución escolar.

Este control es importante, ya que los estudiantes consideran que este tiempo que dedican al videojuego no es excesivo, ni transcendental, esto ocurre porque sus vidas han transcurrido en la era digital, donde la tecnología es muy avanzada y de fácil acceso, algo muy común para ellos. Pero el pasar tanto tiempo usando videojuegos puede ocasionar ciertos trastornos como el sedentarismo, la obesidad, bajas en el rendimiento escolar, evitan los deportes y hasta hacer vida al aire libre. Por otro lado, la investigación detecto que los adolescentes no tienen preferencias marcadas por el tipo de aparato que usan para jugar, puede ser una laptop, el teléfono, 
una consola o la Pc, lo realmente importante es jugar videojuegos, el poder tener acceso a tantos aparatos distintos para jugar, es lo que les permite pasar tanto tiempo usándolos.

Es por ello que se puede afirmar que la influencia de los videojuegos en el comportamiento de los adolescentes estudiantes del 5 to año de bachillerato de la Institución educativa Dr. Jesús María Portillo, no son lo negativas que se pensaría, estos sujetos que se encuentran en la adolescencia intermedia donde ya tienen una noción aproximada de quien son, su identidad y personalidad toman fuerza, es en esta etapa cuando comienzan a adaptarse a otros adolescentes, ellos han podido usar los videojuegos sin presentar los efectos negativos que los autores señalan como consecuencias de su uso, como es la adicción, no se observa que estén aislándose, si bien no comparten tanto tiempo al aire libre, si comparte con amigos y compañeros de clase, por lo menos en el hábito de jugar, presentan una conducta asertiva comunican sus emociones y opiniones, no resuelven sus diferencias de manera violentas, esto pese a que sus videojuegos favoritos son los de acción donde las escenas son de combates, altamente violentas y sangrientas.

\section{Referencias}

Betancourt, R. (2018). Influencia de los videojuegos. Recuperado de https:// www.monografias.com.

Briceño, J; Rojas, D; Chirinos, Y y Alaña, Y. (2018). E-competencias, apropiación social y actitudes hacia las TIC - TAC - TEP en las necesidades formativas del docente. En Chirinos, Ramírez, Barbera, Camejo. Tendencias en la Investigación Universitaria. Una Visión desde Latinoamérica. DOI:www.doi.org/10.47212/ tendencias2018vol.iii.9

Diez, E; Terrón, E y Rojo, J. (2002). Violencia y videojuego. Etic@net. Revista científica electrónica de educación y comunicación en la sociedad del conocimiento. No.0. https:// buleria.unileon.es/bitstream/handle/10612/11826/ViolenciaYVideojuegos6869682.pdf? sequence $=1$ \&isAllowed $=\mathrm{y}$ 
Diccionario de la lengua española. (23. edición). Madrid: Espasa. ISBN 978-84-670-4189-7.

Estallo, J. (1995). Psicopatología y Videojuegos. Editorial Planeta.

Barcelona.

García-Naveira; Alejo; Martin, T; Borja, M; Suárez, A. (2018). Beneficios cognitivos, psicológicos y personales del uso de los videojuegos y sports. Revista de Psicología Aplicada al Deporte y el Ejercicio Físico, vol. 3, núm. 2, 2018, pp. 1-14. https:// www.redalyc.org/jatsRepo/6138/613865230006/613865230006.pdf

Hernández, J. (2015). La influencia de los videojuegos en el proceso de adopción tecnológica. Un estudio empírico en la región de Murcia. Tesis doctoral. Universidad Católica de Murcia.

Hernández, R; Fernández, C y Baptista, P. (2014). Metodología de la investigación. Editorial Mc Graw Hill. España.

Hurtado, J. (2000). Metodología de la investigación. Fundación Sypal. Caracas.

Huizinga, J. (2000). Homo ludens. Alianza editorial. Madrid.

León y López. (2003). Los adolescentes y los videojuegos. Revista apuntes Psicológicos. Universidad de Huelva.

Luzuriaga, J. (2010). Diseño de un protocolo de intervención psicoterapeuta focalizado en la personalidad en adolescentes víctimas de violencia sexual. Tesis de Maestría. Universidad Central del Ecuador.

Madrigales, C. (2012). Autoconcepto en adolescentes de 14 a 18 años (estudio realizado en Funda Niñas Mazatenango). Tesis de Psicología Clínica. Universidad Rafael Landívar. http://biblio3.url.edu.gt/Tesis/2012/05/42/Madrigales-Ceily.pdf 
Marqués, P. (2000). Videojuegos efectos psicológicos. Cuadernos pedagógicos. http:// peremarques.net/videojue.htm

Moncada Jiménez, José; Chacón Araya, Yamileth. (2012). El efecto de los videojuegos en variables sociales, psicológicas y fisiológicas en niños y adolescentes. Retos. Nuevas Tendencias en Educación Física, Deporte y Recreación, núm. 21, enero-junio, 2012, pp. 43

Newman, J. (2004). Videogame. Nueva York. Routledge.

Organización Panamericana de la Salud. (2005). Informe mundial sobre la violencia y la salud para la Organización Mundial de la salud. Washington, D.C.

Papalia, D. (2009). Desarrollo Humano. Mc Graw Hill Internacional. España.

Pérez (2013). Uso y abuso de tecnologías en adolescentes y su relación con algunas variables de personalidad, estilos de crianza, consumo de alcohol y autopercepción como estudiante. Tesis de grado. Universidad de Burgos.

Provenzo, E. (1992). The video generation. The American School Board. Journal.

Silva, I. (2007). La adolescencia y su interrelación con el entorno. Edición Instituto de la juventud. España.

Toyo, Y. (2013). Estudio de las diferentes conductas en la etapa de la adolescencia. Recuperado de https://www.newzoo.com.

Vigueras, E. (2001). El amigo virtual. Recuperado de http:// www.etcetera.com.mx.

Wolf, M. (2001). The medium of the video game. Texas. University of Texas. 